\title{
Nutrition Support in Older Adults
}

\author{
Mette Holst, Ólöf G. Geirsdóttir, and Jack J. Bell
}

\begin{abstract}
Malnutrition is observed in around one in three hospital inpatients; this harmful ICD10-AM-coded disease is a strong independent predictor of adverse older adult and healthcare outcomes, mortality, and treatment costs globally, particularly in multimorbid, older adults. Despite recognition of malnutrition as a disease, nutrition support prescriptions are often not valued as the medicine to treat it. This chapter is devoted to the common nutrition support prescriptions for treating malnutrition: (1) protein- and energy-dense and/or fortified foods, fluids, and menus (HPHE support); (2) oral nutrition supplements; (3) enteral tube feeding; and (4) parenteral nutrition.
\end{abstract}

\footnotetext{
M. Holst

Centre for Nutrition and Intestinal Failure, Aalborg University Hospital, Aalborg University, Aalborg, Denmark

Department of Clinical Medicine, Aalborg University, Aalborg, Denmark e-mail: mette.holst@rn.dk

Ó. G. Geirsdóttir

Faculty of Food Science and Nutrition, School of Health Science, University of Iceland,

Reykjavík, Iceland

e-mail: ogg@hi.is
}

J. J. Bell $(\bowtie)$

Allied Health, The Prince Charles Hospital, Chermside, Queensland, Australia

School of Human Movement and Nutrition Sciences, The University of Queensland, St Lucia, QLD, Australia

e-mail: jack.bell@health.qld.gov.au 


\section{Keywords}

Malnutrition · Nutrition support - Enteral nutrition · Parenteral nutrition · Nutrition assessment

\section{Learning Objectives}

By the end of this chapter, you will be able to:

- Describe the rationale for shared decision-making and informed consent.

- List key questions for older adults and teams to consider prior to commencing, changing, or ceasing nutrition support.

- Apply a systematic approach to nutrition support in older adults.

- Justify the rationale for prescribing HPHE support, oral nutrition supplements, enteral, and parenteral nutrition.

- Value nutrition support actions as medicines prescribed for malnutrition, a harmful, life-limiting disease in older adults.

- Value the need for the involvement of relatives and/or interdisciplinary teams to support nutrition prescriptions in older adults.

\subsection{Nutrition Support in Older Adults: A Hard Edge Best Balanced by Shared Decision-Making, Informed Consent, and Ethical Insight}

Nutrition support (or artificial nutrition) definitions consider care processes provided when unassisted oral intake of food and liquids fails to provide adequate nutrients to meet an individual's estimated requirements [1, 2]. Nutrition support considers prescription of protein- and energy-dense and/or fortified foods, drinks, and menus (HPHE support), oral nutrition supplements (ONS), enteral tube feeding (hereafter enteral nutrition), and parenteral nutrition (PN).

According to the European Society for Clinical Nutrition and Metabolism (ESPEN), artificial nutrition and hydration are a medical intervention; as such these require an indication, a therapeutic goal, the will of the older adult, and consent of the competent older adult or alternative decision-maker [1]. Whether to commence, continue, or withdraw artificial nutrition support in older adults is often a difficult decision. Such decisions should not be made in isolation by a single team member, neither should this be a burden placed on a single older adult or their carer, and may require advice from experts or guiding literature (Chap. 21) [1].

Many guidelines, recommendations, and algorithms for nutrition support exist [1-8]. These often require clinical judgment to apply in real-world individuals across treatment contexts that may be different from where recommendations were developed or considered to be applied. Nutrition support processes can improve 
outcomes for older adults, and their service providers and funders, and are recommended across many settings [4, 8, 9]. However, a balanced approach also should consider the inconsistency of evidence for nutrition support $[10,11]$. There are risks associated with nutrition support, particularly where prescriptions are required to deliver all nutrient and fluid needs; these can be associated with under- or overprescription and/or complications associated with invasive delivery devices that require skilled insertion and careful management. Potential complications associated with the provision of nutrition support are often poorly reported, with a high degree of bias demonstrated across many studies $[4,10]$.

Not surprisingly, given these complexities, interdisciplinary team management of nutrition support is considered best practice. Teams are able to provide expert advice across nutrition care processes, for example, regarding the appropriateness of nutrition support modalities, and recommendations for when it may be appropriate to transition across one or more feeding modalities (Chaps. 6 and 11-13) [4, 12].

What is most important, however, is to include the older adult, or their decisionmaker, in these processes [13]. Shared decision-making is the pinnacle of patientcentered care; Table 5.1 highlights key questions for older adults and teams to consider prior to commencing, changing, or ceasing nutrition support $[1,4$, $9,13-15]$.

Table 5.1 An example nutrition support checklist

- Is there an indication for nutrition support, a therapeutic goal, and informed consent?

-What clinical conditions/comorbidities will influence outcomes?

- Is the older adult actually at risk of refeeding syndrome? If so what do I need to do?

- Is the proposed goal(s) and treatment(s) feasible and realistic?

- Are treating team capabilities and professional and ethical boundaries secured?

- Does this represent ethically appropriate, transparently allocated resources?

-What are the alternative options?

-What is the expected duration and treatment intent of nutrition support?

-What are the risks, burdens, and benefits of the treatment versus no-treatment options?

- How will these influence quality of life or health-related quality of life?

- Is it worth a trial of treatment?

-What are the review timeframes, options, and processes for ongoing care, clinical handover, or discontinuation?

-What are the older adult's treatment preferences and refusal rights?

-What is the older adult's nutrition status and diagnosis(es)?

- Are there any cultural, religious, or legal considerations?

- Does the older adult have adequate health literacy to make an informed consent decision?

- Is it the will of the older adult to proceed?

- Does the older adult have close relatives or carers who are, with consent, informed and encouraged to support nutrition care?

- Are shared goals, decisions, and informed consent processes considered and documented?

- Are nutrition assessment and reassessments, diagnoses, goals, interventions, and monitoring processes appropriately undertaken and documented in line with locally endorsed recommendations, procedures, and/or guidelines?

- Is an interdisciplinary care team organized to support nutrition care across the continuum? 


\subsection{Applying a Systematic Approach to Nutrition Support in Older Adults}

Systematic nutrition care processes should guide delivery of nutrition support. Building on previous chapters and international models, we propose a SIMPLER approach to nutrition support (Table 5.2) $[3,6,7,16]$. We also strongly recommend nutrition support is administered and governed by locally endorsed recommendations, guidelines, and procedures, overseen by nutrition support teams and/or specialists.

\subsection{Protein- and Energy-Dense and/or Fortified Foods, Fluids, and Menus}

Regardless of setting, there is strong consensus that older persons with or at risk of malnutrition should be offered energy- and protein-dense foods and fluids and/or fortified foods; we refer to these as high-protein/high-energy (HPHE) support opportunities. What these look like across global settings are understatedly diverse. These should be texture-modified where oropharyngeal dysphagia and/or chewing problems are evident (Chap. 18) [4]. Additional finger foods or snacks should also be available and offered [4].

A crucial element for HPHE support is accessibility for both the individual and for the care staff that offer them. It is of essential importance that, for example, nutritional drinks and snacks are stored close to the patient and are able to be offered outside of set mealtimes and that these products are cooled or easily heated and completely ready for serving. For people with reduced appetite and dietary intake, it is also important that the portion size is minimized. This may limit the amount of vegetables and fiber consumed. Offering a multivitamin tablet is therefore often necessary for patients who receive "energy- and protein-dense foods," so that the vitamin and mineral needs are optimally covered (Chaps. 2-4). In some care settings, the size of a "small portion" is not implied, and therefore portion sizes may need to be described in detail.

Processes and system opportunities must be leveraged so that the consistency and composition of the diet can be tailored to deliver nutritional needs, both at

Table 5.2 SIMPLER nutrition support processes $[3,6,7,16]$

\footnotetext{
Screen for relevant nutrition diagnoses

Investigate and assess using an ABCDEF approach: anthropometry, biochemistry, clinical, diet and drugs, environment and estimated requirements, function

Make the nutrition diagnosis(es)

Plan with the patient including shared goal setting, informed consent discussion regarding treatment versus no-treatment options, and risks versus benefits implement interventions including nutrition support, education, care integration, and clinical handover Evaluate continuously; ongoing reassessment, monitoring and reviewing of individual patients Review practice through nutrition care process and policy reforms, recommendations, and research
} 
patient population and individual levels accounting for medical, psychosocial, cultural, and environmental factors. Standards and accreditation processes, quality assurance and risk management, policies, funding and resource allocation, industry and political lobbying, and educational, professional, technological, and research processes should all be engaged to ensure high-quality HPHE food choices are readily available to older adults at risk of malnutrition, across the continuum of care. We especially encourage facility administrators to consider the growing evidence base that demonstrates that high-quality menus and foodservice systems improve nutritional intake, the proportion of patients meeting nutritional requirements, and patient satisfaction, while simultaneously reducing food waste and cost [17].

Most importantly, we implore older adults and interdisciplinary teams to identify malnutrition as a harmful disease and value HPHE foods and fluids as the primary medicine to treat it [18].

\subsection{Oral Nutrition Supplements}

Despite dedicated efforts, achieving an adequate protein intake and energy intake through HPHE support alone is not possible, particularly in multimorbid older adults [11]. Where requirements are unlikely to be met, older adults with malnutrition, or those at risk of malnutrition and with chronic conditions, should also be offered ONS, in order to improve dietary intake and body weight and to lower the risk of complications and readmission [4].

ONS are available in different flavors and are energy and protein rich; most also provide additional micronutrients. These drinks routinely have a long shelf life and so can be consumed as a snack when off the ward or out of the house. Where ONS are offered, there is strong consensus that these provide at least $400 \mathrm{kcal} / \mathrm{day}$, including $30 \mathrm{~g}$ or more of protein/day, and continue for 4 weeks or more [4]. When ONS are offered to an older person, recommendations support continuing for at least 1 month, with ongoing monthly assessment of efficacy and expected benefit [4].

At times older adults may be unable to meet their protein and energy requirements despite HPHE support and ONS prescription, due to the reduced desire or ability to consume or absorb nutrients and/or increased requirements of acute or chronic conditions. In such cases enteral or parenteral tube feeding may be indicated [1].

\subsection{Artificial Tube Feeding}

Nutrition support, in particular EN and PN, are considered as medical treatments rather than basic cares. Consequently, these should only be applied in situations where there is a realistic chance of improving or maintaining the older adult's condition and quality of life [1,4]. In older adults with inadequate nutritional intake in the terminal or palliative phase of illness, comfort feeding should be offered [4]. Other circumstances, for example, where risks are considered to 
outweigh the expected benefits, will demand interdisciplinary assessment and clinical judgment, and determining older adult wishes and realistic treatment goals, noting that sometimes trials of tube feeding may be appropriate to consider $[1,4]$. In the following sections, we provide an overview of EN and PN. However, we refer the reader to locally endorsed policies, procedures, and guidelines that must be enacted and audited to adequately govern artificial tube feeding in older adults.

\subsubsection{Enteral Nutrition}

In older persons with active treatment goals, enteral tube feeding (enteral nutrition (EN)) should be considered where oral intake is expected to be below half of energy requirements for more than 1 week, or absent for more than 3 days, despite attention to promote oral intake adequacy [4]. Where EN aligns with shared treatment goals, this should be initiated without delay [4]. The ongoing benefits and potential risks of EN require regular assessment, reassessment, and monitoring processes directed by locally endorsed procedures and guidelines. A review should also be undertaken whenever there is a change in condition or treatment planning [4]. Wherever possible and safe to do so, oral intake should still be encouraged throughout the duration of EN.

\subsubsection{Feeding Tube Types}

Depending on physiological, physical, environmental, and personal factors, feeding tubes can be via nasogastric, nasojejunal, orogastric, orojejunal, gastrostomy, or jejunal routes. The most common are the nasogastric tubes (NGT) and PEG (percutaneous endoscopic gastrostomy), both of which feed directly into the stomach. With lifelong tube feeding, a low-profile balloon gastrostomy, or PEG button, may be used, which is less obtrusive to the older adult. The button probe also empties into the stomach. In addition, there are also probes that empty further into the intestine, for example, PEGJ probes (PEG with jejunal tube extension). Alternatively, a tube may be entered directly through the abdomen at the jejunum (e.g., surgical jejunostomy). A typical course is to start with the insertion of a nasogastric feeding tube through the nose, and later, if the need for prolonged tube feeding is assessed, a longer-term feeding device is implanted.

\subsubsection{Tube Placement}

Installation of naso-enteral feeding tubes should be performed by those trained in the procedure; this is usually a nurse or medical professional. Preparation and involvement of the older adult, good time, and a calm environment are important. Complications to placement due at times occur, for example, malpositioning into the lungs or accidental removal. In many settings, X-ray control is prescribed, where the guide wire in the nasal probe clarifies the location of the probe. There may be local agreements regarding control of location, where X-ray control is deselected, for example, in very restless patients/cerebrally affected patients. 
The PEJ catheter or the PEGJ probe can be inserted in connection with a surgical operation. PEG, PEGJ, and button probe insertions require a scopic procedure when tube feeding is needed over a longer period of time, i.e., more than 3 months. There is a small risk of intestinal perforation or other complications during construction; although rare, these may have life-threating or ending consequences [19]. These should be inserted by appropriate trained specialists.

It is necessary for the nurse to make sure that the written prescriptions are in place and that the location of the probe after construction has been seen and approved by the medical professional before starting the nutrition.

\subsubsection{Tube Considerations and Care Requirements}

There are many considerations regarding tube care and requirements; institutions must have local guidelines/procedures and access to experts who may assist or give advice regarding management and care requirements for tubes [20]. It is important that the nurse, dietitian, or other accountable healthcare provider is aware of these local procedures for identifying the type of probe, the site of insertion and the place of discharge, and management processes. For example, PEG versus PEGJ tubes may look similar. However, while bolus feeding via PEGs is generally acceptable, PEGJs should not be used for bolus administration of tube feeding as it empties into the middle of the small intestine and not into the ventricle. Similarly, when "exercising" probes as part of good practice management, some probes, for example, PEGs, may require rotating, while rotating PEGJs is routinely not recommended. Leaving nasogastric tubes in situ for extended periods without appropriate management practices may lead to a pressure injury. Similarly, gastrostomy insertion sites should be observed for signs of infection; older adults should be provided with management education where appropriate.

\subsubsection{Checking Placement}

Before each administration of nutrition, fluid or medicine, ensure the probe is positioned correctly in line with local standards or recommendations. Commonly recommended methods to check positioning include reading the markings on the probe at the insertion site and/or $\mathrm{pH}$ values of aspirates. In many settings it is suggested to start with slow administration, for example, with $20-30 \mathrm{~mL}$ of water, where no resistance may be experienced; the patient should be observed for signs of misplacement, such as cough, nausea, and respiratory effects.

\subsubsection{Flushing Tubes}

Tubes should be flushed regularly. Local guidelines and fluid and medication requirements will all need to be considered when determining individual flush volumes and frequencies, for example, four hourly flushes for continuous feeds with at least $30 \mathrm{~mL}$, flushes of water before and after medication, before and after intermittent or bolus feedings, and when pump feeds are commenced or stopped. Flushing the tube promptly after gastric aspiration for $\mathrm{pH}$ testing, but not before, is often recommended. It may be appropriate to provide increased or additional water flushes or to meet fluid requirements. It is commonly recommended for continuous 
pump feeds to use the Y port on the feeding tube when flushing to maintain a closed system. Disconnecting the feeds to flush is not recommended. Where a tube is not actively being used for feeding purposes, for example, during trials of oral intake, or is being used for alternative purposes, local guidelines for flushing should be considered.

\subsubsection{Feeding Regimens and Modality}

Dependent on settings, dietitians or medical staff prepare an individual tube feeding plan, which is usually prescribed and documented in the medical record. Several sections of hospitals have also prepared guiding nutritional instructions, which contain a standard start-up of tube nutrition. In this way, the nurses can ensure the patient a faster start-up of tube feeding, which can later be adapted by a dietitian or medical nutrition specialist. For patients in need of enteral nutrition, several different types of feeding tubes can be constructed.

The choice feeding modality should consider the older adult's individual needs and preferences with the aim to provide safe enteral nutrition and hydration appropriate to the clinical status, in addition to functional and quality of life issues [15]. Optimal feeding times and modality remain inconclusive; we would recommend considering local, national, and/or disease-specific recommendations when choosing feeding modality. Options include, for example, pump versus gravity feeding or bolus, continuous, intermittent, or overnight feeding approaches. Which method chosen should consider the advantages and disadvantages of each mode of feeding, interdisciplinary team recommendations, clinical judgment, treatment goals, additional therapies and medications, and what matters to the older adult.

There are a broad variety of enteral tube feeding preparations available globally; product-specific recommendations are beyond the remit of this text. The final product prescribed is guided by individual requirements, evidence-based recommendations for macro- and micronutrients, costs, logistics, tube feeding hardware (e.g., pumps, giving sets), care provider contracts, and purchasing agreements.

\subsubsection{Formula Handling and Management}

When setting up and dismantling, local hygiene measures must always be observed, as tube feeding must be considered as a food that can be contaminated by improper handling. The manufacturer's product instructions should always be observed. All feeds should be cross-checked according to local prescribing practices and visually inspected, and the use by date noted prior to administration. Aseptic technique should always be applied when handling feeds. Medications, water, dye, or pancreatic/biliary fluids should not be added enteral nutrition formulas. Hang times should be governed by both the product description and local practice requirements. Local practices should also be in place for what is required when breaking ready-to-hang, "closed" systems.

\subsubsection{Parenteral Nutrition (PN)}

$\mathrm{PN}$ is a form of nutrition support administered through an intravenous (iv) approach. Where aligned with treatment goals and expected benefit, recommendations support 
offering older adults PN where combined oral and enteral tube intake is expected to be impossible for more than 3 days or below half of energy requirements for more than 1 week.

Parenteral nutrition is a prescription drug. The nutrition plan is often planned by the medical professional in consultation with the dietitian and nurse, with consideration to the individual's fluid, macronutrient (energy, protein, fat, carbohydrate), and micronutrient requirements. Addition of vitamins and minerals must be performed under aseptic conditions or provided separately.

Parenteral nutrition often has a slow administration time of 10-16 h. Setup time should ideally be planned accounting for the patient's daily rhythm and other treatment requirements. Administration of parenteral nutrition can be given briefly, as full or supplementary nutrition, or for some older adults for life, for example, for those with short bowel syndrome. While central iv accesses are predominantly applied in most settings, for short durations or where central access is not an option, parenteral nutrition can also be set up using peripheral access. There is generally a difference in the concentration of preparations for use via central and peripheral routes; these are routinely highlighted on the products for administration. In case of lifelong administration, the parenteral nutrition can be given at home under the guidance and control of nutrition support teams or services. Other patient groups with gastrointestinal obstruction may also be discharged with parenteral nutrition to the home for longer or shorter periods, but routinely with a requirement for hospital or specialist outpatient review. Knowledge of the work around parenteral nutrition has also become a relevant area for the nurse specialists in addition to dietitians and medical professionals.

While interdisciplinary management of PN is globally considered best practice, how this "looks," what will be prescribed, who will prescribe it, and how this will be monitored will vary according to health service resources and infrastructure, specialist staff availability, patient populations and treatment settings, clinical and professional governance, and sociocultural factors. We strongly recommend that PN is administered and audited in line with locally endorsed policies, procedures, and guidelines that must be in place to govern safe, effective parenteral nutrition.

\subsection{Preventing and/or Managing Nutrition Support Complications}

\subsubsection{Medications}

Medications, whether ONS, EN, PN, or other medications and pharmaceutical products, should always be administered according to local guidelines and medication-specific recommendations. These should account for food-drug and drug-nutrient interaction (Chap. 20). Many medicines, in addition to prescribed tube feeds, can be administered through EFT devices. However, the administering healthcare professional must always check what is appropriate to administer via EN or PN tubes, for example, whether current tablets are appropriate to be crushed and given via an NGT. We recommend discussion with local pharmacist and treating medical professional and setting specific recommendations. 


\subsubsection{Aspiration}

Underlying illnesses, acute complications, procedures, medications, changing levels of consciousness, feeding tube (mis)placement, and feeding positions are all risk factors for aspiration. Interdisciplinary teams should screen for dysphagia and implement safe swallowing strategies and modified diets where indicated and appropriate (Chap. 18). Dedicated attention to oral hygiene and mouthcares may reduce the risk of aspiration pneumonia. For patients receiving enteral tube feeding, a check should be undertaken to confirm that the feeding tube is in the proper position before initiating feedings. Elevating the head of bed for individuals on bedrest should be considered, both during and for a period after feeding, ensuring positioning and supported to prevent sliding down in bed and creating shear forces [12]. Although evidence is limited, avoiding excessive feeding tube rates or bolus volumes and considering continuous pump feeding over bolus feeding where patients have demonstrated intolerance to gastric bolus feeding may be appropriate [12, 21-23]. Where high risk of aspiration, demonstrated intolerance to gastric feeding or in older adults with demonstrated reflux and aspiration of gastric contents, jejunal feeding should be considered [12]. Recent consensus guidelines also suggest avoiding supplementary overnight tube feeding in older adults with a hip fracture due to lack of beneficial effects, patient burden, and poor tolerance [4]. All efforts should be made to minimise use of sedation and prevent delirium $[4,12]$ the administration of gastric enteral feedings. The relative risks and benefits of promotility agents should also be considered [12].

\subsubsection{Gastrointestinal Upset}

Diverse factors influence gastrointestinal status. Patients receiving nutrition support are often observed to experience constipation, diarrhea, or gastrointestinal obstruction; in many cases these are independent of the nutrition support approach prescribed, for example, underlying illness or disease, infections (e.g., Clostridium difficile), antibiotics, and other medications. Medication review should consider both potential causes (e.g., antibiotics, magnesium, laxatives) and prescription of antiemetics (vomiting) or medications to manage diarrhea. For patients with ongoing vomiting, a reduced pump feeding rate or change to continuous feeding may be appropriate if the patient is receiving bolus feeding. For patients with bolus feeding, it should be confirmed that the feeding device is in the gastric ventricle. For all patients with severe or prolonged gastrointestinal upset, it is important to assess whether the patient is receiving adequate fluid to maintain fluid balance.

\subsubsection{Delayed Gastric Emptying or Gastroparesis}

Numerous medications impair gastric emptying, particularly opiates, some antidepressants, and dopamine. Again, the relative risks and advantages of gastric emptying agents/prokinetics should be considered. Post-pyloric feeding should also be considered where ongoing. Although recommendations and practices for 
monitoring gastric residual volumes vary widely, recommendations support avoiding withholding EN for gastric residual volumes (GRVs) $<500 \mathrm{~mL}$ in the absence of other signs of intolerance and implementing measures to reduce risk of aspiration for volumes between 250 and $500 \mathrm{~mL}$ [12].

\subsubsection{Hyperglycemia}

Where hyperglycemia is observed, medical staff should be informed prior to changing nutrition support prescriptions. Hyperglycemia is commonly observed in acutely unwell, multimorbid older adults; opportunities for medical management, rather than restricting dietary or tube feeding intake, should be carefully considered by the treating team particularly in older adults with demonstrated inadequate intake or malnutrition. This approach is supported by recent guidelines strongly recommending that in older patients with diabetes mellitus, malnutrition and risk of malnutrition shall be managed according to recommendations for malnourished older persons without diabetes mellitus [4].

\subsubsection{Refeeding Syndrome}

There is limited consensus regarding the diagnosis and management strategies for refeeding syndrome $[12,24]$. Recent consensus guidelines recommend starting early and gradually increasing EN or PN in malnourished older adults in 3 days, supplementing thiamine even in the case of suspected mild deficiency, while monitoring and correcting blood levels of phosphate, magnesium, and potassium and glucose $[4,12]$. We recommend referring to locally endorsed guidelines for refeeding management.

\subsection{Summary}

Nutrition support in older adults is often complex and is best overseen by interdisciplinary nutrition support teams. However, the daily work of delivering nutrition support is routinely undertaken by the older adults themselves, family and friends, nurses, and other interdisciplinary healthcare providers. Shared goal setting and decision-making is the pinnacle of patient-centered care. We implore healthcare providers to work together with older adults to identify malnutrition as a harmful, life-limited disease that may be treated with nutrition support, wherever this aligns with what matters to the older adult.

\section{Take-Home Points}

- Artificial nutrition and hydration are a medical intervention; as such these require an indication, a therapeutic goal, the will of the patient, and consent of the competent patient or alternative decision-maker.

- Patients and interdisciplinary teams must identify malnutrition as a harmful disease and value HPHE foods and fluids as the primary medicine to treat it. 
- Where requirements are unlikely to be met, older adults with malnutrition, or those at risk of malnutrition and with chronic conditions, should be offered ONS.

- In older persons with active treatment goals, enteral tube feeding should be considered where oral intake is expected to be below half of energy requirements for more than 1 week or absent for more than 3 days.

- Where aligned with treatment goals and expected benefit, older adults should be offered PN where combined oral and enteral tube intake is expected to be impossible for more than 3 days or below half of energy requirements for more than 1 week.

\section{References}

1. Druml C, Ballmer PE, Druml W, Oehmichen F, Shenkin A, Singer P et al (2016) ESPEN guideline on ethical aspects of artificial nutrition and hydration. Clin Nutr 35(3):545-556

2. National Institute for Health and Clinical Excellence (NICE) (2006) Nutrition support in adults: oral nutrition support, enteral tube feeding and parenteral nutrition (clinical guideline 32)

3. Bell JJ, Young A, Hill J, Banks M, Comans T, Barnes R et al (2018) Rationale and developmental methodology for the SIMPLE approach: a Systematised, Interdisciplinary Malnutrition Pathway for impLementation and Evaluation in hospitals. Nutr Diet 75(2):226-234

4. Volkert D, Beck AM, Cederholm T, Cruz-Jentoft A, Goisser S, Hooper L et al (2019) ESPEN guideline on clinical nutrition and hydration in geriatrics. Clin Nutr 38(1):10-47

5. Volkert D, Beck AM, Cederholm T, Cereda E, Cruz-Jentoft A, Goisser S et al (2019) Management of malnutrition in older patients-current approaches, evidence and open questions. J Clin Med 8(7):974

6. Swan WI, Vivanti A, Hakel-Smith NA, Hotson B, Orrevall Y, Trostler N et al (2017) Nutrition care process and model update: toward realizing people-centered care and outcomes management. J Acad Nutr Diet 117(12):2003-2014

7. Cederholm T, Barazzoni R, Austin P, Ballmer P, Biolo G, Bischoff SC et al (2017) ESPEN guidelines on definitions and terminology of clinical nutrition. Clin Nutr 36(1):49-64

8. Mueller C, Compher C, Ellen DM (2011) A.S.P.E.N. clinical guidelines: nutrition screening, assessment, and intervention in adults. J Parenter Enter Nutr 35(1):16-24

9. Tappenden KA, Quatrara B, Parkhurst ML, Malone AM, Fanjiang G, Ziegler TR (2013) Critical role of nutrition in improving quality of care: an interdisciplinary call to action to address adult hospital malnutrition. J Parenter Enter Nutr 37(4):482-497

10. Feinberg J, Nielsen EE, Korang SK, Halberg Engell K, Nielsen MS, Zhang K et al (2017) Nutrition support in hospitalised adults at nutritional risk. Cochrane Database Syst Rev 5:CD011598

11. Stratton RJ, Elia M (2007) A review of reviews: a new look at the evidence for oral nutritional supplements in clinical practice. Clin Nutr Suppl 2(1):5-23

12. Boullata JI, Carrera AL, Harvey L, Escuro AA, Hudson L, Mays A et al (2017) ASPEN safe practices for enteral nutrition therapy [Formula: see text]. JPEN J Parenter Enteral Nutr 41(1): 15-103

13. King PC, Barrimore SE, Pulle RC, Bell JJ (2019) "I Wouldn't Ever Want It": a qualitative evaluation of patient and caregiver perceptions toward enteral tube feeding in hip fracture inpatients. J Parenter Enter Nutr 43(4):526-533

14. Barry MJ, Edgman-Levitan S (2012) Shared decision making - the pinnacle of patientcentered care. N Engl J Med 366(9):780-781

15. Mon AS, Pulle C, Bell J (2018) Development of an 'Enteral tube feeding decision support tool' for hip fracture patients: a modified Delphi approach. Aust J Ageing 37(3):217-223

16. Bell JJ, Geirsdóttir ÓG, Hertz K, Santy-Tomlinson J, Skúladóttir SS, Eleuteri S et al (2020) Nutritional care of the older patient with fragility fracture: opportunities for systematised, interdisciplinary approaches across acute care, rehabilitation and secondary prevention settings. In: Orthogeriatrics. Springer, Cham, pp 311-329 
17. McCray S, Maunder K, Barsha L, Mackenzie-Shalders K (2018) Room service in a public hospital improves nutritional intake and increases patient satisfaction while decreasing food waste and cost. J Hum Nutr Dietet 31(6):734-741

18. Bell JJ, Bauer J, Capra S, Pulle CR (2013) Barriers to nutritional intake in patients with acute hip fracture: time to treat malnutrition as a disease and food as a medicine? Can J Physiol Pharmacol 91(6):489-495

19. Milanchi S, Wilson MT (2008) Malposition of percutaneous endoscopic-guided gastrostomy: guideline and management. J Minim Access Surg 4(1):1-4

20. ESPEN Webinar Enteral accesses for enteral nutrition in adults . Nutrition E. Enteral Nutrition, Adults and Adolescents (>15 yr) (2021). https://www.google.com/search?rlz=1C1GCEA_ enIS778IS778\&sxsrf=ALeKk03SJdw8ztTt7iOiQgPqyzRkci_3g:1625224667813\&q=Nutriti on+E.+Enteral+Nutrition,+Adults+and+Adolescents+(\%3E15+yr) $+2021 ;($ November+2018) .\&spell=1\&sa=X\&ved=2ahUKEwiegIXYocTxAhUygv0HHcGsBDUQBSgAegQIARAz\&bi $\mathrm{w}=1280 \& \mathrm{bih}=577$. November 2018.

21. Bischoff SC, Austin P, Boeykens K, Chourdakis M, Cuerda C, Jonkers-Schuitema C et al (2020) ESPEN guideline on home enteral nutrition. Clin Nutr 39(1):5-22

22. Gkolfakis P, Arvanitakis M, Despott EJ, Ballarin A, Beyna T, Boeykens K et al (2021) Endoscopic management of enteral tubes in adult patients - Part 2: Peri- and post-procedural management. European Society of Gastrointestinal Endoscopy (ESGE) Guideline. Endoscopy 53(2):178-195

23. Torsy T, Saman R, Boeykens K, Duysburgh I, Van Damme N, Beeckman D (2018) Comparison of two methods for estimating the tip position of a nasogastric feeding tube: a randomized controlled trial. Nutr Clin Pract 33(6):843-850

24. Matthews-Rensch K, Capra S, Palmer M (2021) Systematic review of energy initiation rates and refeeding syndrome outcomes. Nutr Clin Pract 36(1):153-168

\section{Recommended Reading}

Bell JJ, Young AM, Hill JM, Banks MD, Comans TA, Barnes R, Keller HH (2021) Systematised, Interdisciplinary Malnutrition Program for impLementation and Evaluation (SIMPLE) delivers improved hospital nutrition care processes and patient reported experiences - an implementation study. Nutr Diet. https://doi.org/10.1111/1747-0080.12663

Boullata JI, Carrera AL, Harvey L, Escuro AA, Hudson L, Mays A et al (2017) ASPEN safe practices for enteral nutrition therapy [Formula: see text]. JPEN J Parenter Enteral Nutr 41(1):15-103

Druml C, Ballmer PE, Druml W, Oehmichen F, Shenkin A, Singer P et al (2016) ESPEN guideline on ethical aspects of artificial nutrition and hydration. Clin Nutr (Edin, Scot) 35(3):545-556

King PC, Barrimore SE, Pulle RC, Bell JJ (2019) "I Wouldn't Ever Want It": a qualitative evaluation of patient and caregiver perceptions toward enteral tube feeding in hip fracture inpatients. J Parenter Enter Nutr 43(4):526-533

Volkert D, Beck AM, Cederholm T, Cruz-Jentoft A, Goisser S, Hooper L et al (2019) ESPEN guideline on clinical nutrition and hydration in geriatrics. Clin Nutr 38(1):10-47

Open Access This chapter is licensed under the terms of the Creative Commons Attribution 4.0 International License (http://creativecommons.org/licenses/by/4.0/), which permits use, sharing, adaptation, distribution and reproduction in any medium or format, as long as you give appropriate credit to the original author(s) and the source, provide a link to the Creative Commons license and indicate if changes were made.

The images or other third party material in this chapter are included in the chapter's Creative Commons license, unless indicated otherwise in a credit line to the material. If material is not included in the chapter's Creative Commons license and your intended use is not permitted by statutory regulation or exceeds the permitted use, you will need to obtain permission directly from the copyright holder. 